\title{
Protein tyrosine phosphatase receptor type D (PTPRD)-mediated signaling pathways for the potential treatment of hepatocellular carcinoma: a narrative review
}

\author{
Xuejing Huang, Feizhang Qin, Qiuhua Meng, Min Dong \\ School of Pharmacy, Guangxi Medical University, Nanning, China \\ Contributions: (I) Conception and design: M Dong, X Huang; (II) Administrative support: M Dong; (III) Provision of study materials or patients: M \\ Dong; (IV) Collection and assembly of data: X Huang, F Qin, Q Meng; (V) Data analysis and interpretation: M Dong, X Huang; (VI) Manuscript \\ writing: All authors; (VII) Final approval of manuscript: All authors. \\ Correspondence to: Min Dong. School of Pharmacy, Guangxi Medical University, Nanning, China. Email: dongmin0217@sina.com.
}

\begin{abstract}
Hepatocellular carcinoma (HCC) is the third most common cause of cancer-related mortality worldwide, and the methods for its treatment have shown limited efficacy. There is an urgent need to explore the underlying mechanism that are involved in hepatocarcinogenesis, contributing to find various signal molecular targets for HCC diagnosis, prevention and therapy. Recently, Various studies have illustrated protein tyrosine phosphatase receptor type D (PTPRD) is an important tumor-suppressor gene that is down-regulated in HCC and this downregulation occurs through its promoter hypermethylation. PTPRD is involved in the progression, migration, apoptosis, invasion and immune suppression of HCC. Also, PTPRD participates in several vital cellular signaling pathways, including PTPRD, signal transduction and activation of transcription 3 (STAT3), JAK, PTPRD, $\beta$-catenin, TCF, along with the PTPRD-CXCL8 axis, the PTPRD/phosphatidylinositol3-kinase (PI3K)/mammalian target of rapamycin (mTOR), and the PTPRD/ PD-1/programmed death receptor ligand-1 (PD-L1) axis, thus playing an essential role in HCC. Therefore, PTPRD can be considered as a novel therapeutic target for HCC, and PTPRD-targeted therapeutics in combination with methylation inhibitors, immune checkpoint inhibitors and alternative targeted drugs maybe an innovative treatment method for HCC. However, clinical research of PTPRD-targeted therapies in HCC is greatly limited and tremendous efforts are strongly urged to evaluate its clinical performance in HCC. In this review, we summarized the physiological function and the significant effects of PTPRD and performed a comprehensive analysis of PTPRD-targeted strategies for HCC.
\end{abstract}

Keywords: Protein tyrosine phosphatase receptor type D (PTPRD); signal transduction and activation of transcription 3 (STAT3); hepatocellular carcinoma (HCC); signaling pathway

Submitted May 15, 2020. Accepted for publication Aug 18, 2020.

doi: $10.21037 /$ atm-20-4733

View this article at: http://dx.doi.org/10.21037/atm-20-4733

\section{Introduction}

Hepatocellular carcinoma (HCC) is the most prevalent primary liver cancer and is considered the sixth most prevalent cancer, and the second leading cause of cancer mortality worldwide (1).

Many factors can cause and accelerate the progression of this chronic malignancy, including metabolic diseases and other risk factors like hepatitis B virus (HBV), hepatitis
$\mathrm{C}$ virus (HCV), alcohol abuse, and inflammatory liver disease (2). As advanced-stage HCC patients are often diagnosed too late, most cases are generally treated by surgical resection or percutaneous and transarterial interventions. However, these therapeutic methods have limited efficacy. Worse still, chemotherapy and radiotherapy offer an inferior prognosis, and involve serious adverse reactions and a poor survival rate, leaving this disease 
with no effective therapeutic strategy (3). There is an urgent need for assessing new molecular targets as a novel diagnostic and therapeutic option for HCC, confronted with these obstacles. It is understood that many oncogenic factors cause the emergence and development of HCC, but the underlying molecular mechanism is still largely unclear.

Recently, protein tyrosine phosphatase receptor type D (PTPRD) has been discovered as a promising therapeutic target owing to PTPRD inactivation in several malignancies (4). A previous study found that the deactivation of PTPRD is relevant to many genetic and epigenetic abnormalities, like gene amplification and DNA methylations, Along with chromosomal anomalies, such as chromosomal deletion, rearrangement, and euploidy. Furthermore, according to the level of PTPRD mRNA expression, PTPRD is significantly decreased in liver cirrhosis and HCC samples and is typically expressed in healthy liver samples (5). There is a significant difference between normal samples and HCC samples, according to their PTPRD expression profile. Moreover, there is a tendency towards a lower expression of PTPRD in cancer samples with higher grades compare to lower grades (5). These findings indicate PTPRD may function as a tumor-suppressor gene. The tumor-suppressive properties of PTPRD are mainly associated with signal transduction and activation of transcription 3 (STAT3). Previous studies have confirmed that constitutive activation of STAT3 frequently leads to p-STAT3 overexpression while PTPRD is significantly downregulated in HCC (6), showing that PTPRD and STAT3 have a significant association with HCC.

Furthermore, substantial evidence indicates PTPRD negatively regulates the STAT3-mediated signaling pathway by directly dephosphorylating STAT3 at Y705 and prohibiting dimerization and nuclear translocation of STAT3 (7). Thus, PTPRD can disrupt the negative regulators of STAT3 to inhibit its oncogenic functions. Cell signaling pathways are involved in regulating important cellular processes, including cell proliferation, cell cycle progression, apoptosis, and autophagy. Considering the relationship between PTPRD and STAT3, we hypothesize PTPRD participates in the STAT3-mediated signaling pathways to control STAT3 carcinogenicity. Besides, PTPRD also play an important role in several vital cellular signaling pathways in HCC, such as PTPRD/Wnt/ $\beta$-catenin/TCF, The PTPRD-CXCL8 axis, PTPRD/ phosphatidylinositol3-kinase (PI3K)/Akt/mammalian target of rapamycin (mTOR) and PTPRD/PD-1/programmed death receptor ligand-1 (PD-L1) axis etc. On this basis, the PTPRD- mediated signaling pathway are currently being widely explored to develop novel therapies for HCC. Generally, PTPRD expression may have potential clinical value, and PTPRD can be regarded as a promising STAT3 inhibitor, a better cancer biomarker, and a novel therapeutic target that may improve the therapeutic efficacy of HCC. In this review, we introduce the structure, functionality, and the physiological role of PTPRD in human cancers, and summarize the underlying signal transduction pathway of PTPRD. We emphasize PTPRD's role as a potential target for anticancer therapeutic interventions and hope to give credibility to the hypothesis that PTPRD is a strong HCC tumor-suppressing gene.

We present the following article in accordance with the Narrative Review reporting checklist (available at http:// dx.doi.org/10.21037/atm-20-4733).

\section{The structure of PTPRD}

PTPRD was first explored in chickens and flies in 1990, and the analysis of human RNA sequencing data has discovered PTPRD is mostly expressed in the brain (RPKM 18.0), kidney (RPKM 8.8), and 14 other tissues. PTPRD is a transmembrane protein located in $9 \mathrm{p} 23-$ 24.1(at chromosome9-NC_000009.12) (8). It consists of an extracellular region, which possesses three Ig-like and eight fibronectin type III-like domains, a single transmembrane region, and two tandem intracytoplasmic catalytic domains referred to as D1 and D2 (9) and the structure of PTPRD was showed in Figure 1. PTPRD's physiological functions depend on its D1 domain that mediates phosphates activity of cytoplasmic proteins, while the D2 domain is mostly enzymatically inactive although it shares sequence homology with phosphates. Furthermore, the D2 domains can downregulate the activity of the D1 domain. Classical PTPRD is composed of 1,912 amino acids, with an approximate mass of $215 \mathrm{kDa}(9)$.

The structure of STAT3 consists of six conserved domains: a C-terminal transactivation domain, a Src homology region 2 (SH-2) domain, a linker domain, a DNA-binding domain, a coiled-coil domain, and an $\mathrm{N}$-terminal domain (NH2). The mechanism by which PTPRD regulates STAT3 involves the transactivation domain of STAT3, which contains two phosphorylation $(\mathrm{P})$ sites: tyrosine residue $(\mathrm{Y})$ at 705 and 727 . Once STAT3 is activated, the $\mathrm{P}(\mathrm{Y})-\mathrm{SH}-2$ domains interact to form a STAT3 dimmer. As showed in Figure 1, PTPRD negatively regulates the STAT3-mediated signaling pathway by its 


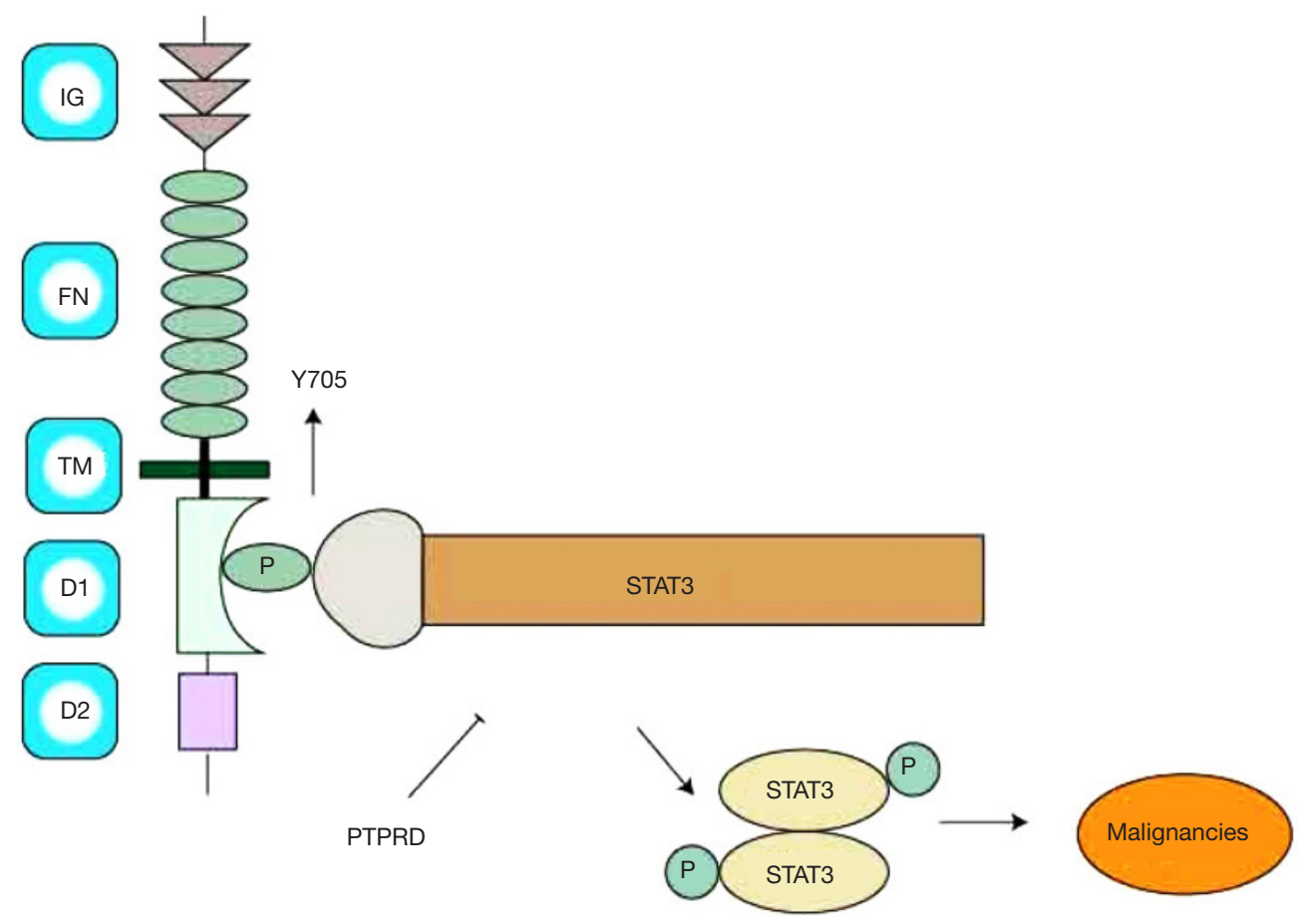

Figure 1 The structure of protein tyrosine phosphatase receptor type D (PTPRD) consisting of three immunoglobulin (Ig) like (IG) and eight fibronectin type II-like domains (FN), a single transmembrane region (TM), and two tandem intracytoplasmic catalytic domains: D1 and D2.

D1 domain directly dephosphorylating STAT3 at Y705 and prohibiting dimerization and nuclear translocation of STAT3. So we speculate that PTPRD might attenuate STAT3 phosphorylation activity that contributes to potentially suppress HCC.

\section{The functions of PTPs}

PTPRD belongs to the PTP family, which has a total of 107 members. PTPs are regarded as signaling molecules that regulate various cellular processes, including cell proliferation, differentiation, mitotic cycle, and migration.

To our knowledge, PTPs can be broadly divided into receptor-like forms and non-receptor forms. Non-receptor PTPs contain one domain that mediates protein-protein interactions: Src-homology2 (SH2). The receptor-like PTPs consists of a single transmembrane domain, and variable extracellular domains that are related to cell adhesion, with the intracellular components containing two tandem PTP domains: D1 and D2 (8). PTPs play a part in the signaling mechanism. It is accepted PTPs are involved in protein dephosphorylation via the addition of a phosphate group onto a Tyr residue (pTyr), Ser residue(pSer), or Thr residue (pThr), which play a crucial part in regulating signal transduction.

PTPs participate in the significant regulation of cell adhesion and ligand recognition, serving as the stimulation threshold. Recent research has reported that several PTPs could downmodulate $\mathrm{T}$ cell activation and were associated with autoimmunity. For example, SHP2 is recruited to TCR microclusters and inhibits the signaling of PD-L1 ligands, though, for instance, the dephosphorylation of ZAP70, and prevents CD28/PKC $\theta$ association. It is tempting to hypothesize that PTPs could passively mediate the PD-1 signaling pathway (10). PTPRD is a member of the leukocyte common antigen-related protein (LAR) subfamily of receptor-type protein tyrosine phosphates (PTPs).

The LAR family has three members in humans: PTPRF, PTPRD, and PTPRS. Several studies have indicated PTPRD is capable of directly interacting with PTPRS, and the SLIT- and NTRK-like (SLITRK) proteins also can bind to PTPRD, leading to the accumulation of vesicular gamma-aminobutyric acid (GABA) transporter (as known as a marker for synaptic specialization), which suggests PTPRD functions as a ligand for neuronal cell adhesion (9). Besides this, many studies have revealed PTPRD knockout may be a marker for cognitive impairment, and can cause 
Table 1 An overview of the effects of PTPRD on various cancers

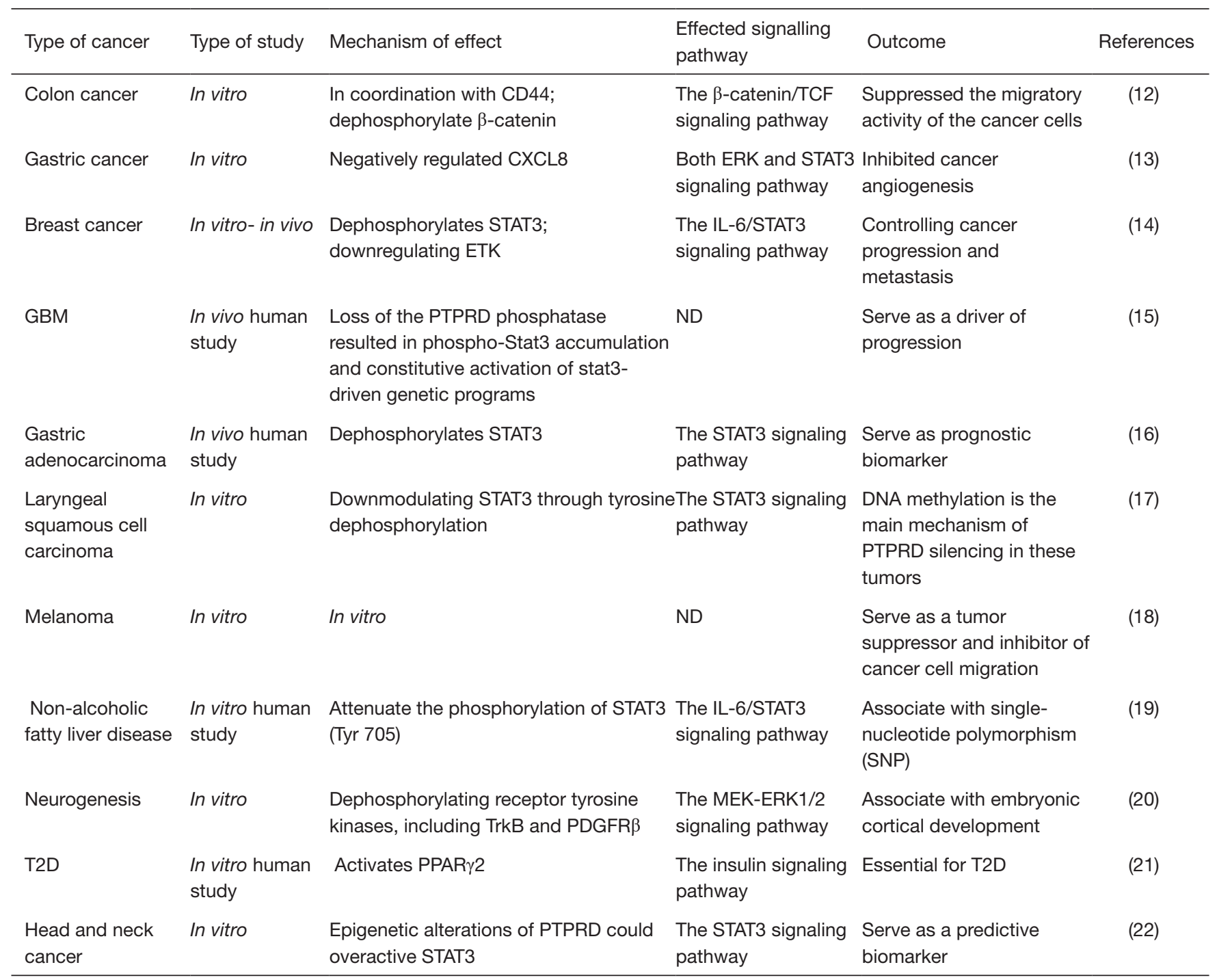

PTPRD, protein tyrosine phosphatase receptor type D; GBM, glioblastoma multiforme; ND, not defined; TCF, T-cell factor; T2D, type 2 diabetes.

hearing loss, hyposensitivity to diabetes, and increase the risks of hypertension (11). The findings reveal the critical effects that altered PTPRD expression can have on the human nervous, endocrine, and cardiovascular systems. Similarly, research has found that deletion, mutation, copy number loss, and hypermethylation of PTPRD can lead to various cancers (Table 1), which indicates PTPRD may be a significant tumor-suppressor gene (12-22).

\section{The functions of PTPRD in HCC}

In summary, under physiological conditions, PTPRD exerts various functions that inhibits HCC cell migration and invasion in vitro, whereas knockdown of PTPRD significantly promotes invasion and metastasis. PTPRD becomes persistently inactivated in the majority of malignancies, PTPRD is significantly down-regulated in HCCs, and its expression negatively is associated with genetic and epigenetic alterations. Notably, PTPRD deficiency is significantly correlates with pathogenesis, shorter survival, poor prognosis and increased recurrence in patients with HCC. PTPRD-regulated HCC invasiveness is accompanied by changes of STAT3, JAK, $\beta$-catenin, TCF, along with the PTPRD-CXCL8 axis, the PTPRD/ 
$\mathrm{PI} 3 \mathrm{~K} / \mathrm{mTOR}$, and the PTPRD/PD-L1 axis, PTPRD might dephosphorylate those signal molecules and inactivates their oncoprotein that regulates related signal pathway, and mediates tumor migration, invasion, and metastasis. It substantiates the clinical significance of PTPRD in HCC. We hypothesize PTPRD as a tumor suppressor candidate and strategies designed to activate or upregulate PTPRD might provide a promising treatment method for HCC.

\section{The PTPRD mediated signaling pathways in HCC}

It is well accepted that tyrosine phosphorylation is of great importance in the overall process of intracellular signal transduction. Tumor cells are confronted with sustained stimulation from a variety of cytokines and signal transduction molecules, such as STAT3, JAK, $\beta$-catenin, CXCL8 (C-X-C motif ligand 8), mTOR, PD-L1, and so forth. The pathways mediated by those factors are all up-regulated during HCC progression. PTPRD plays an indispensable role in the regulation of above signal transduction molecules to block their phosphorylation cascades might potentially inhibited HCC progress. Here, we summarize the potential roles of PTPRD in the regulation of the cell signalling pathways.

\section{PTPRD/STAT3/FAK}

STAT3 has recently emerged as a potential therapeutic target for HCC due to its crucial roles in oncogenesis (23). It belongs to the STAT family of cytoplasmic transcription factors that mediate signal transduction from the plasma membrane to the nucleus in various cellular activities. Several signaling molecules primarily activate STAT3, including ligand binding to G-protein coupled receptors (GPCRs), toll-like receptors (TLRs), cytokine receptors, growth factor receptors, and non-receptor tyrosine kinases (24).

Physiologically, a cytokine [such as interleukin-6 (IL-6)] binding to its specific cell surface receptor initiates a conformational change in the receptor, which in turn leads to recruitment and activation of the associated Janus kinases (JAKs). Activated JAKs then phosphorylate specific residues on the intracellular domains of the cytokine receptor, which serve as docking sites for associated STAT proteins. Upon docking with the activated receptor-associated JAK phosphorylate STATs, the phosphorylated STATs dissociate from the receptor chains, dimerize, and translocate to the cell nucleus, where they activate gene transcription (25). Growing evidence has shown that overexpression and aberrant activation of STAT3 has been reported to be closely related to pathogenesis of HCC, and patients with HCC exhibit nuclear STAT3 in tumoral but not surrounding non-tumoral tissues. In addition, a significant association has been found between STAT3 activation and clinical pathological features including larger tumor size, vascular invasion, advanced stage in patients with HCC (24), indicating that under pathological conditions, some cytokines arouse constitutive activation of JAK/STAT3 signal pathway that may promote HCC proliferation. One of the central mechanisms that negatively regulate STAT3 activation is dephosphorylation of the tyrosine residue essential for its activation by PTPs. As mentioned above, PTPRD acts as a tumor suppressor via its ability to negatively regulate STAT3-mediated signaling (26). In patients with HCC, high PTPRD levels coincide with a decrease in the expression levels of phosphorylated STAT3, a decrease in tumor recurrence after surgical resection, and an increase of patient survival from HCC (26). Thus, PTPRD potentially has a negative feedback loop to regulate IL-6/STAT3/JAK signaling. PTPRD decreases STAT3 phosphorylation and prevents pSTAT3 from translocating into the nucleus, thus leading to the downregulation of the signaling molecules, including MMP-2 and MMP-9, that promote proliferation and angiogenesis of cancer cells. Taken together, the above shows that a PTPRD/JAK/STAT3 axis may potentially be the therapeutic point for HCC.

\section{PTPRD/Wnt/B-catenin/TCF}

It is widely acknowledged the aberrant activation of the canonical $\mathrm{Wnt} / \beta$-catenin/TCF signaling pathway causes liver cancer. $\beta$-catenin is a transcription cofactor that has a critical influence on the Wnt signaling pathway. As soon as Wnt combines with the heterodimeric receptor complex of a frizzled $(\mathrm{Fz})$ transmembrane lipoprotein receptor-related protein (LPR), Wnt/ $\beta$-catenin signaling is deactivated. As a consequence, non-phosphorylated active $\beta$-catenin accumulates in the cytoplasm and translocates into the nucleus where it binds to the T-cell factor (TCF). Then, TCF regulates the expression of target genes involved in HCC, like CD44, cyclin D1, c-Myc, and others. One study confirmed mutations of the $\mathrm{N}$-terminal region of $\beta$-catenin contribute to liver carcinogenesis (27), which suggests $\beta$-catenin is an oncogene of HCC. When $\beta$-Catenin/TCF signaling is activated, CD44 expression is enhanced. Preclinical studies have found that positive cooperation exists between PTPRD and CD44, as both 
jointly regulate cell migration and progression (12). PTPRD interacts with cell-adhesion molecules, including $\beta$-Catenin and E-cadherin, through its D2 domain, and PTPRD dephosphorylates $\beta$-Catenin causing $\beta$-catenin/ TCF signaling to suppress cancer cell migration, thereby stabilizing cell adhesion and inhibiting cancer cell migration (12). In addition to this, current evidence suggests the leukocyte common antigen-related protein (LAR), a receptor-like PTP, has a role in cadherin complexes where it associates with and dephosphorylates $\beta$-Catenin (28).

As mentioned above, PTPRD belongs to the LAR family, so it supports a function for PTPRD in the regulation of epithelial cell-cell contacts at adherens junctions and in the control of $\beta$-catenin signaling. It is, therefore, possible that $\beta$-catenin is a critical transcriptional activator of PTPRD, and the suppression of PTPRD leads to the disruption of the $\beta$-catenin/E-cadherin complex and cell-cell adhesion. In summary, as the activation of the canonical $\mathrm{Wnt} / \beta$-catenin signaling pathway is related to abnormal activity of $\beta$-catenin in HCC, and PTPRD negatively controls $\beta$-Catenin, the PTPRD/Wnt/ $\beta$-catenin/TCF signaling pathway may thus serve as an attractive target for developing novel rational therapies for HCC.

\section{The PTPRD-CXCL8 axis}

Proinflammatory cytokines, being secreted in the tumor microenvironment, is crucial in the development and metastasis of HCC. C-X-C motif ligand 8 (CXCL8), also known as interleukin-8 (IL-8), is a chemokine secreted by tumor cells in an autocrine or paracrine manner and serves as a significant multifunctional cytokine to modulate tumor proliferation, invasion, and migration. CXCL8 belongs to the CXC family, which plays an essential physiological role in tissue homeostasis, cell recruitment, and activation (29). When the epidermal growth factor (EGF) binds to epidermal growth factor receptor (EGFR), this can trigger the activity of the Ras/Raf/MEK/ERK pathway to stimulate tumor cells to produce various inflammatory factors, like CXCL5, CXCL8, IL-6, and IL-1 in the patients with HCC (30).

The overexpression of CXCL8 is closely associated with intrahepatic metastasis, tumor size, and tumor stage of HCC (30), and CXCL8 is suspected to promote HCC proliferation, invasion, and metastasis. In addition to this, another study has demonstrated the levels of the transcription factor forkhead box C1 (FOXC1) and CXCL8 were increased in HCC tissues compared with adjacent non-tumor liver tissues and that patients with the co-overexpression of FOXC1 and CXCL8 had worse outcome and poorer prognosis than those with lower levels. FOXC1 stimulates the secretion of CXCL8, associated with activation of PI3K/ Akt/HIF- $\alpha$ signaling pathway (31), suggesting that CXCL8 might be an adverse prognostic factor, oncogene, and biological marker of HCC. Previous studies have revealed that a significant increase in CXCL8 expression and PTPRD was frequently deactivated in gastric cancer and that PTPRD silencing leads to the upregulation of CXCL8, p-STAT3, and p-ERK, which indicates PTPRD might negatively regulate CXCL8 through decreasing the phosphorylation of ERK and STAT3 (13). The negative regulation further implicates PTPRD as a potential regulator of CXCL8 signaling, and, as CXCL8 can promote HCC development and might be the downstream molecule of PTPRD, we hypothesize PTPRD possibly inhibits the CXCL8-mediated signaling pathway and that the PTPRD-CXCL8 axis may also serve as a potential therapeutic strategy for HCC.

As shown in the Figure 2, The combination of three different mechanisms of PTPRD- mediated signaling pathways in HCC: (I) PTPRD can suppress the phosphorylation of STAT3 (on Tyr 705) and the subsequent STAT3 nuclear translocation to the downregulation of the IL-6/JAK/ STAT3 signal transduction pathways in HCC. (II) PTPRD dephosphorylates $\beta$-catenin and negatively regulates $\beta$-catenin/TCF signaling and its target CD44 to suppress the migration of HCC. (III) PTPRD is a potential regulator of CXCL8 and reduces its expression to inhibit the carcinogenic effect of CXCL8 in HCC. As a result, STAT3, $\beta$-catenin and CXCL8 proteins are phosphorylated in a signal-dependent manner by PTPRD leads to the downregulation of their expression in HCC.

\section{PTPRD/PI3K/Akt/mTOR}

mTOR is a serine/threonine kinase that plays an essential role in modulating metabolic pathways for cell proliferation, growth, and metabolism, as well as the regulation of autophagy. The mTOR gene is located in chromosome 1 (1p36.22) and belongs to a protein family called PI3Krelated kinase (PIKK), which forms the core of two distinct multiprotein signaling complexes: mTOR complex 1 (mTORC1) and mTOR complex 2 (mTORC 2) (32). When $G$ protein combines with its receptor, the activated $G$ proteins dissociate into $G a$ subunits and $G \beta-\gamma$ ligand complexes.

After this, it recruits and activates phosphatidylinositol 3 kinase- $\gamma$ (PI3K- $\gamma$ ). The activation of PI3K triggers the production of phosphatidylinositol-3,4,5-triphosphate 


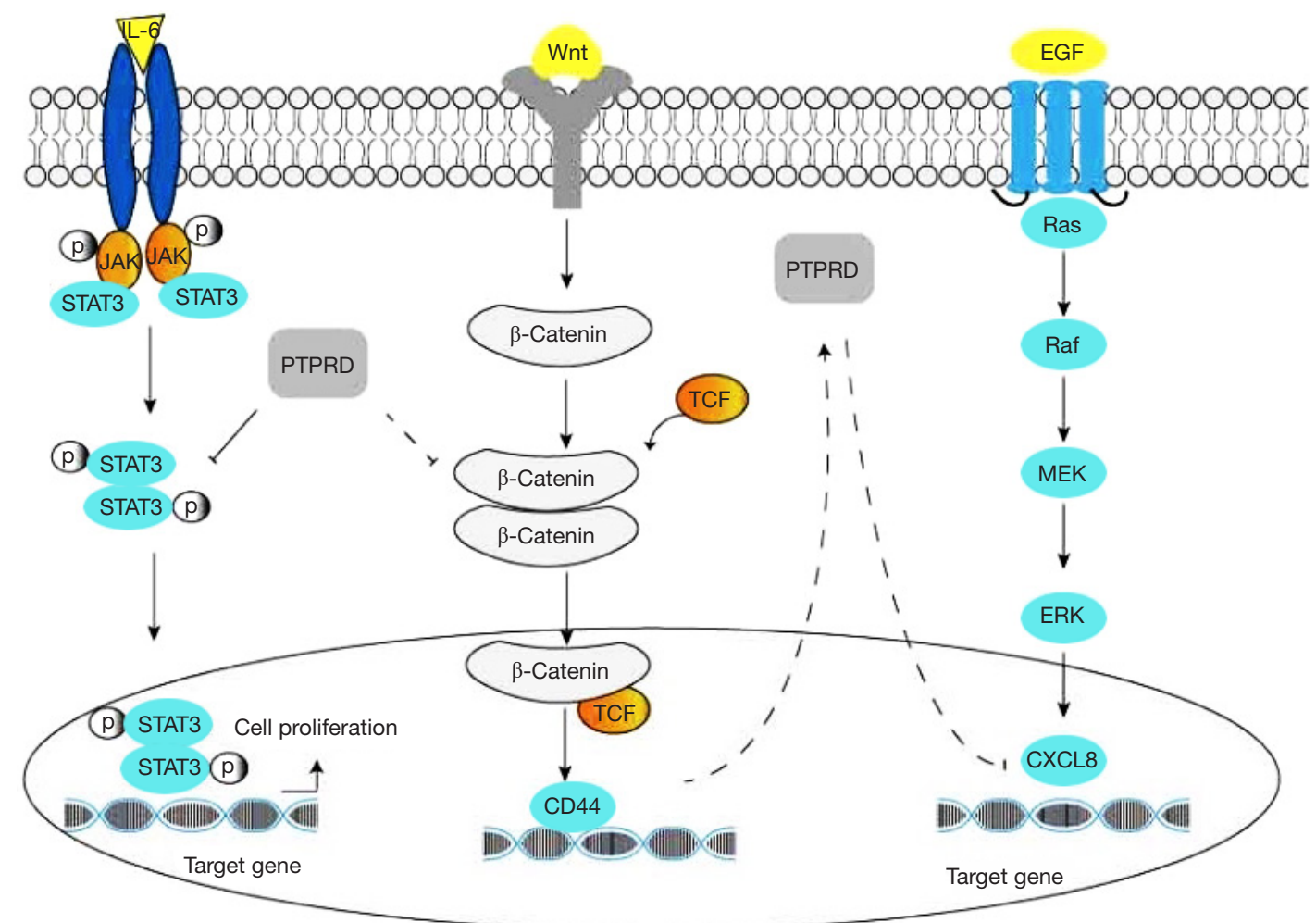

Figure 2 The mechanism of the protein tyrosine phosphatase receptor type D (PTPRD) regulation of the signaling pathway: PTPRD can suppress the phosphorylation of signal transduction and activation of transcription 3 (STAT3) (on Tyr 705) and the subsequent STAT3 nuclear translocation to the downregulation of the IL-6/JAK/STAT3 signal transduction pathways in hepatocellular carcinoma (HCC). PTPRD dephosphorylates $\beta$-catenin and negatively regulates $\beta$-catenin/TCF signaling and its target CD44 to suppress the migration of HCC. PTPRD is a potential regulator of CXCL8 and reduces its expression to inhibit the carcinogenic effect of CXCL8 in HCC.

(PIP3) by phosphorylating phosphatidylinositol-4,5bisphosphate (PIP2). Then, PIP3 as a second messenger, induces protein kinase B (Akt), and, following activation, AKT can catalyze downstream effector proteins like mTOR to promote several cellular processes. By inhibiting the phosphorylation of PIP3, the phosphatase and tensin homolog (PTEN) protein acts as a negative regulator of the PI3K/Akt pathway.

A previous study revealed that after mTOR inhibitors were employed, the levels of mTOR and STAT3 decreased significantly compared to the control group, which shows STAT3 is the downstream target molecule of mTOR (33). It is well-known that the PI3K /Akt/mTOR pathway is one of the most deregulated signaling pathways in HCC. PTPRD has been found to negatively regulate STAT3, suggesting that PTPRD has an anticancer effect via the suppression of $\mathrm{PI} 3 \mathrm{~K} / \mathrm{Akt} / \mathrm{mTOR}$ signaling pathway to restrict HCC cell migration and invasion. Again, this is another avenue by which PTPRD can be used to treat HCC.

\section{The PTPRD/PD-1/PD-L1 axis}

Immunotherapy is considered the major modern breakthrough in oncology and has already become the most successful class of treatment for cancers due to its efficacy and safety. It has been firmly established $\mathrm{T}$ cells can recognize and kill antigen-expressing cells directly and integrate adaptive and innate effector mechanisms to orchestrate diverse immune responses. However, the immunosuppressive tumor microenvironment also generates a vast immune imbalance, leading to an insufficient immune response and the uncontrollable proliferation of cancer cells. Notably, cancer cells not only directly suppress anticancer immune mechanisms, but also cause immune cells to induce and maintain an immunosuppressive microenvironment. Tumors are able to escape the host immune response by 


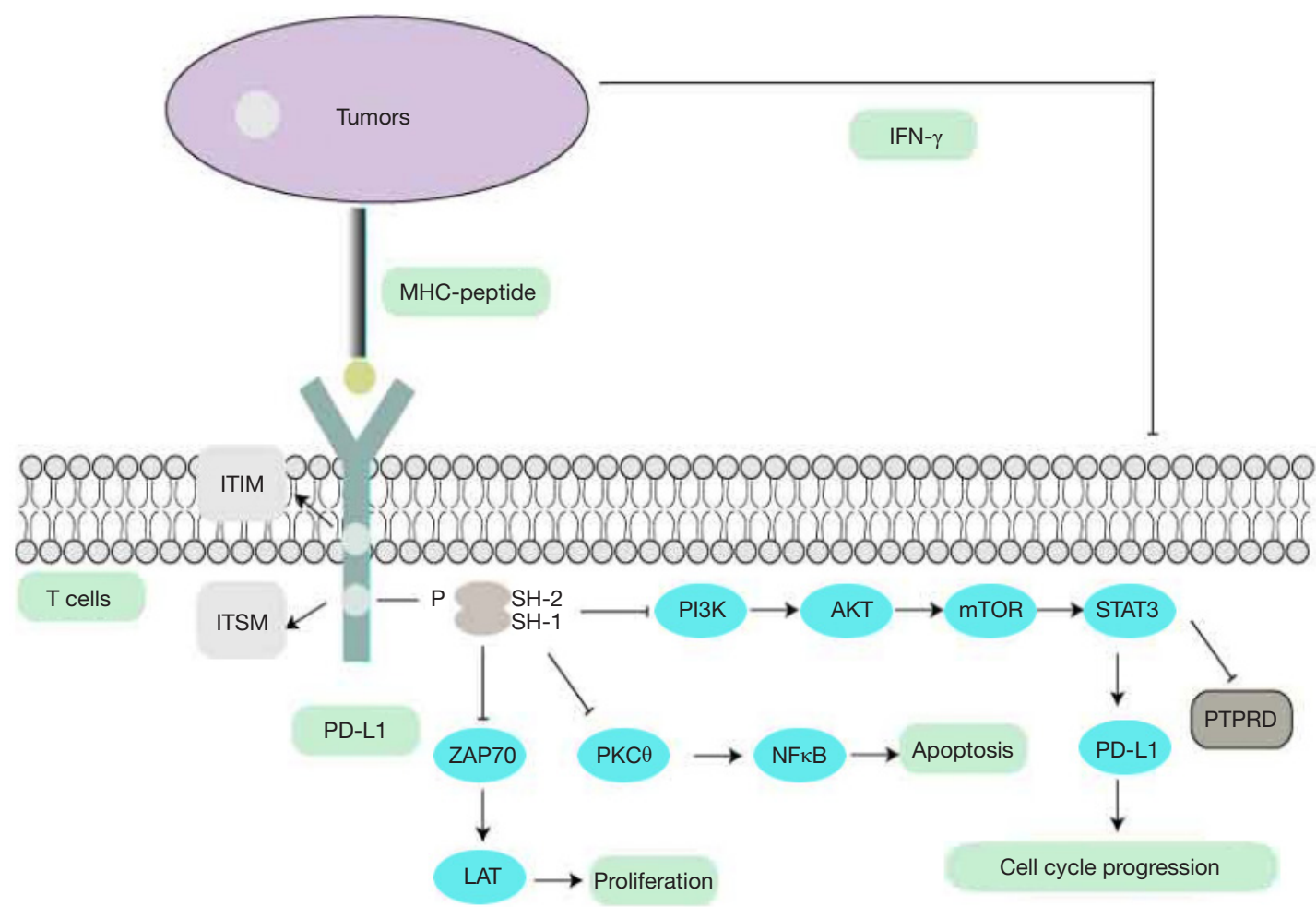

Figure 3 Protein tyrosine phosphatase receptor type D (PTPRD) negatively regulates PI3K/AKT/mTOR signaling pathway and reduces signal transduction and activation of transcription 3 (STAT3) phosphorylation, resulting in the downregulation of programmed death receptor ligand-1 (PD-L1).

promoting the expression of chemokines (such as IL-10), checkpoints cytotoxic T lymphocyte antigen-4 (CTLA-4) and programmed death-ligand 1 (PD-1) (34).

Immunotherapy impedes the pathways that inhibit immune cell activation and stimulate immune responses against tumor cells (35). Among various immunotherapy checkpoints, programmed cell death protein-1 (PD-1, also called CD279) and its ligand PD-L1 axis have played an important role in many cancer treatments. The PD-1 is a 50-55-kDa type I transmembrane glycoprotein located on chromosome 2q.37.3, and belongs to the immunoglobulin (Ig) superfamily. PD-1 consists of an Ig-V-like extracellular domain, a transmembrane domain, and a cytoplasmic domain that harbors tyrosine-based signaling inhibitory motifs (ITIM) and an immune receptor tyrosine-based switch motif (ITSM) (Figure 3). These are separate potential phosphorylation sites and are involved in the activity of $\mathrm{T}$ cells. SHP-1 and SHP-2 can bind to the ITSM, which leads directly to the dephosphorylation of molecules that are downstream of the TCR signaling to inhibit cell survival gene Bcl-XL, decrease interleukin-2 (IL-2) production, and prolonged glucose metabolism. Downregulation of IL-2 requires further induction of T cells inactivation. Furthermore, it is well-established that protein PTEN can inhibit the activity of PI3K, while PD-L1 could trigger Akt signaling activation to inhibit $\mathrm{T}$ cell proliferation.

PD-1 interacts with two ligands, PD-L1 (CD274) and PD-L2 (CD273). The expression of PD-L1 can be detected in the hematopoietic cells which include $\mathrm{T}$ cells, B cells, macrophages, and non-hematopoietic healthy tissue cells like vascular endothelial cells, keratinocytes, and placenta syncytiotrophoblast cells (36). The PD-1/ PD-L1 axis plays an essential role in eliciting the immune checkpoint response of $\mathrm{T}$ cells to suppress $\mathrm{T}$ cell migration, proliferation, and secretion of cytotoxic mediators, which then induce cytokine deletion. Therefore, tumor cells are capable of evading immune surveillance. Therefore, blocking the integration of PD-1 and PD-L1 can partly reduce the effect of tumor immune escape and rescue the cytotoxic cell-induced immune response. Recently, HCC patients were demonstrated expressing high levels of PD-L1, which contributes to be at a significantly higher risk 
of cancer recurrence, metastasis, and cancer-related death. On the above facts, the PD-1/PD-L1 axis has proven to be a relatively effective treatment strategy for HCC (36).

The immune system mostly regulates immune cells to maintain physiological balance. As shown in the Figure 3, in the tumor microenvironment, antigenpresenting cells (APCs) can recognize the neo-antigens that arise from the surface of cancer cells. APCs then present the antigen to the immature $T$ cells via $\mathrm{T}$-cell receptors, after which the activated $\mathrm{T}$ cells recognize the tumor antigens and begin to attack tumor the cells. These cells secrete interferon-gamma (IFN- $\gamma$ ) to stimulate cancer cell-expressing PD-1and PD-L1, resulting in positive cancer cells acquiring immune tolerance. And it could be one of many mechanisms responsible for PD-L1 upregulation in cancer cells. Furthermore, activation of common oncogenic pathways like JAK/ STAT/Ras/Erk/EMT, or PI3K/AKT/mTOR $(37,38)$, along with treatment with cytotoxic agents, has also been shown to affect tumoral PD-L1 expression. For example, STAT3 has also been demonstrated to bind to the PD-L1 promoter to transcriptionally regulate $\mathrm{PD}-\mathrm{L} 1$ expression, which may promote tumor immune evasion in HCC (39). As previously mentioned, STAT3 is a substrate of PTPRD, and PTPRD has a negative feedback loop to regulate the STAT3-mediated signaling pathway. We can, therefore, speculate that PTPRD is a negative regulator of the STAT3-mediated transcription of PD-L1 in various cancers. Based on the above research, we propose a hypothesis that PTPRD might restrain the activation of prosurvival pathways and the transcriptional factors, STAT3, by inhibiting its phosphorylation. The inhibiting leads to the suppression of PD-L1 expression, involved in the antitumor immune response of malignant tumors. Therefore, it is suggested that PTPRD possibly key to understanding the mechanism that regulates the expression of PD-L1 and may be critical for HCC immunotherapy.

\section{The future perspectives of PTPRD-targeted therapies in HCC}

At present, sorafenib, a multikinase inhibitor, is the only systemic first-line therapy option for advanced HCC cases. However, patients treated with multikinase inhibitors received insufficient survival benefit, and thus there remain urgent needs in advanced-stage patients. Proper combinative targeted therapy may improve the prognosis of advanced HCC. Therefore, combination therapy is a promising treatment modality for HCC. The PTPRDmediated signaling pathway has emerged as a promising target for the treatment of HCC.

On the evidence discussed above, we propose a series of hypotheses: (I) as far as PTPRD itself is concerned, PTPRD is a potential tumor suppressor gene for HCC. DNA's methylations contribute to the inactivation of PTPRD, but methylation inhibitors can be used to prevent the methylation of PTPRD from restoring its physiological function and cancer-suppressing effect, although its efficient delivery and stability in vivo remain technically challenging. PTPRD combined with methylation inhibitors may be a new treatment for HCC. (II) Resistance to therapy frequently occurs in advanced HCC, and a combined approach may be more effective than monotherapy. STAT3, mTOR, and PD-1/PD-L1 are carcinogenic molecules, and the application of STAT3-targeted, mTOR-targeted, PD-1/ PD-L1-targeted drugs may be a promising therapeutic approach against HCC. As potential downstream molecules of PTPRD, PTPRD downregulates them to decrease their expression level in HCC. We posit that in combination strategies, PTPRD can be considered a negative mediator to suppress the activity of STAT3/JAK, $\beta$-catenin/TCF, the PTPRD-CXCL8 axis, and PI3K/mTOR/STAT3. PTPRD, in conjunction with other anticancer therapeutics, (including STAT3 inhibitors and mTOR inhibitors), might be a better biomarker strategy to improve the therapeutic efficacy of HCC. (III) Nivolumab, a small molecule immune checkpoint that targets PD-1/PD-L1, has a manageable safety profile, and durable objective responses show the potential of nivolumab for the treatment of advanced HCC (40). Thus, strategies to combine PTPRD with immune checkpoint inhibitors that reactivate immune responses from suppression may have considerable potential and clinical therapeutic value.

\section{Conclusions}

PTPRD is a tumor-suppressor gene that has a crucial role in the initiation, promotion, and progression of HCC. The evidence suggests that a gradual loss of PTPRD expression is a common event in liver disease progression, and it highlights the role of PTPRD as a potential suppressor of hepatocarcinogenesis, irrespective of etiology. Many studies have been conducted concerning the pathological role of PTPRD and its pivotal contribution to the regulation of signaling pathways, including those of STAT3/JAK, $\beta$-catenin/TCF, the PTPRD-CXCL8 axis, the PI3K/Akt/ 
mTOR axis, and the PD-1/PD-L1 axis. Current evidence demonstrates that a remarkable relationship exists between PTPRD expression and signaling networks, which operates to regulate the tumorigenesis and progression of cancer cells. Hence, PTPRD, in combination with current chemotherapy, can be regarded as a novel therapeutic target for the treatment of HCC. However, further investigation is needed to determine the therapeutic potential of PTPRD in HCC therapy.

\section{Acknowledgments}

The authors thank Min Dong for providing help and support. Min Dong initiated and coordinated the whole study, participated in the design of the study, drafting the manuscript and data interpretation. All authors have read and approved the final manuscript.

Funding: This work was support by National Natural Science Foundation of China (No. 81302859); Guangxi Natural Science Foundation (No. 2018GXNSFAA050121; 2017GXNSFAA198145).

\section{Footnote}

Reporting Checklist: The authors have completed the Narrative Review reporting checklist. Available at http:// dx.doi.org/10.21037/atm-20-4733

Conflicts of Interest: All authors have completed the ICMJE uniform disclosure form (available at http://dx.doi. org/10.21037/atm-20-4733). The authors have no conflicts of interest to declare.

Ethical Statement: The authors are accountable for all aspects of the work in ensuring that questions related to the accuracy or integrity of any part of the work are appropriately investigated and resolved.

Open Access Statement: This is an Open Access article distributed in accordance with the Creative Commons Attribution-NonCommercial-NoDerivs 4.0 International License (CC BY-NC-ND 4.0), which permits the noncommercial replication and distribution of the article with the strict proviso that no changes or edits are made and the original work is properly cited (including links to both the formal publication through the relevant DOI and the license). See: https://creativecommons.org/licenses/by-nc-nd/4.0/.

\section{References}

1. Bray FI, Ferlay J, Soerjomataram I, et al. Global cancer statistics 2018: GLOBOCAN estimates of incidence and mortality worldwide for 36 cancers in 185 countries. CA Cancer J Clin 2018;68:394-424.

2. Yang JD, Hainaut P, Gores GJ, et al. A global view of hepatocellular carcinoma: trends, risk, prevention and management. Nat Rev Gastroenterol Hepatol 2019;16:589-604.

3. Boland P, Wu J. Systemic therapy for hepatocellular carcinoma: beyond sorafenib. Chin Clin Oncol 2018;7:50.

4. Uhl GR, Martinez MJ, Paik P, et al. Cocaine reward is reduced by decreased expression of receptor-type protein tyrosine phosphatase $\mathrm{D}$ (PTPRD) and by a novel PTPRD antagonist. Proc Natl Acad Sci U S A 2018;115:11597-602.

5. Acun T, Demir K, Oztas E, et al. PTPRD is homozygously deleted and epigenetically downregulated in human hepatocellular carcinomas. OMICS 2015;19:220-9.

6. Chan TA, Heguy A. The protein tyrosine phosphatase receptor $\mathrm{D}$, a broadly inactivated tumor suppressor regulating STAT function. Cell Cycle 2009;8:3063-4.

7. Tang L, Liu JX, Zhang ZJ, et al. High expression of Anxa2 and Stat 3 promote progression of hepatocellular carcinoma and predict poor prognosis. Pathol Res Pract 2019;215:152386.

8. Ostman A, Hellberg C, Böhmer FD. Proteintyrosine phosphatases and cancer. Nat Rev Cancer 2006;6:307-20.

9. Uhl GR, Martinez MJ. PTPRD: neurobiology, genetics, and initial pharmacology of a pleiotropic contributor to brain phenotypes. Ann N Y Acad Sci 2019;1451:112-29.

10. Saito T, Yokosuka T, Hashimototane A. Dynamic regulation of $\mathrm{T}$ cell activation and co-stimulation through TCR-microclusters. FEBS Lett 2010;584:4865-71.

11. Choucair N, Mignonravix C, Cacciagli P, et al. Evidence that homozygous PTPRD gene microdeletion causes trigonocephaly, hearing loss, and intellectual disability. Mol Cytogenet 2015;8:39.

12. Funato K, Yamazumi Y, Oda T, et al. Tyrosine phosphatase PTPRD suppresses colon cancer cell migration in coordination with CD44. Exp Ther Med 2011;2:457-63.

13. Bae WJ, Ahn JM, Byeon HE, et al. PTPRD-inactivationinduced CXCL8 promotes angiogenesis and metastasis in 
gastric cancer and is inhibited by metformin. J Exp Clin Cancer Res 2019;38:484.

14. Yu X, Zhang F, Mao J, et al. Protein tyrosine phosphatase receptor-type $\delta$ acts as a negative regulator suppressing breast cancer. Oncotarget 2017;8:98798-811.

15. Veeriah S, Brennan C, Meng S, et al. The tyrosine phosphatase PTPRD is a tumor suppressor that is frequently inactivated and mutated in glioblastoma and other human cancers. Proc Natl Acad Sci U S A 2009; 106:9435-40.

16. Wang D, Wang L, Zhou J, et al. Reduced expression of PTPRD correlates with poor prognosis in gastric adenocarcinoma. PLoS One 2014;9:e113754.

17. Szaumkessel M, Wojciechowska S, Janiszewska J, et al. Recurrent epigenetic silencing of the PTPRD tumor suppressor in laryngeal squamous cell carcinoma. Tumour Biol 2017;39:1010428317691427.

18. Walia V, Prickett TD, Kim JS, et al. Mutational and functional analysis of the tumor-suppressor PTPRD in human melanoma. Hum Mutat 2014;35:1301-10.

19. Nakajima S, Tanaka H, Sawada K, et al. Polymorphism of receptor-type tyrosine-protein phosphatase delta gene in the development of non-alcoholic fatty liver disease. J Gastroenterol Hepatol 2018;33:283-90.

20. Tomita H, Cornejo F, Arandapino B, et al. The Protein Tyrosine Phosphatase Receptor Delta Regulates Developmental Neurogenesis. Cell Rep 2020;30:215.

21. Chen YT, De Lin W, Liao WL, et al. PTPRD silencing by DNA hypermethylation decreases insulin receptor signaling and leads to type 2 diabetes. Oncotarget 2015;6:12997-3005.

22. Peyser ND, Du Y, Li H, et al. Loss-of-Function PTPRD Mutations Lead to Increased STAT3 Activation and Sensitivity to STAT3 Inhibition in Head and Neck Cancer. PLoS One 2015;10:e0135750.

23. Lee C, Cheung ST. STAT3: An Emerging Therapeutic Target for Hepatocellular Carcinoma. Cancers (Basel) 2019;11:1646.

24. Attili I, Karachaliou N, Bonanno L, et al. STAT3 as a potential immunotherapy biomarker in oncogeneaddicted non-small cell lung cancer. Ther Adv Med Oncol 2018;10:1758835918763744.

25. Owen KL, Brockwell NK, Parker BS. JAK-STAT Signaling: A Double-Edged Sword of Immune Regulation and Cancer Progression. Cancers (Basel) 2019;11:2002.

26. Ortiz B, Fabius AW, Wu WH, et al. Loss of the tyrosine phosphatase PTPRD leads to aberrant STAT3 activation and promotes gliomagenesis. Proc Natl Acad Sci U S A 2014;111:8149-54.

27. Wang W, Smits R, Hao H, et al. Wnt/ $\beta$-Catenin Signaling in Liver Cancers. Cancers (Basel) 2019;11:926.

28. Mooney RA, LeVea CM. The leukocyte common antigen-related protein LAR: candidate PTP for inhibitory targeting. Curr Top Med Chem 2003;3:809-19.

29. Ha H, Debnath B, Neamati N. Role of the CXCL8CXCR1/2 Axis in Cancer and Inflammatory Diseases. Theranostics 2017;7:1543-88.

30. Huang P, Xu X, Wang L, et al. The role of EGFEGFR signalling pathway in hepatocellular carcinoma inflammatory microenvironment. J Cell Mol Med 2014;18:218-30.

31. Huang W, Chen Z, Zhang L, et al. Interleukin-8 Induces Expression of FOXC1 to Promote Transactivation of CXCR1 and CCL2 in Hepatocellular Carcinoma Cell Lines and Formation of Metastases in Mice. Gastroenterology 2015;149:1053-67.

32. Rebouissou S, Nault J. Advances in molecular classification and precision oncology in hepatocellular carcinoma. J Hepatol 2020;72:215-29.

33. Hossan MS, Chan ZY, Collins HM, et al. Cardiac glycoside cerberin exerts anticancer activity through PI3K/AKT/mTOR signal transduction inhibition. Cancer Lett 2019;453:57-73.

34. Thomson AW, Knolle PA. Antigen-presenting cell function in the tolerogenic liver environment. Nat Rev Immunol 2010;10:753-66.

35. Topalian SL, Drake CG, Pardoll DM. Immune Checkpoint Blockade: A Common Denominator Approach to Cancer Therapy. Cancer Cell 2015;27:450-61.

36. Li K, Tian H. Development of small-molecule immune checkpoint inhibitors of PD-1/PD-L1 as a new therapeutic strategy for tumour immunotherapy. J Drug Target 2019;27:244-56.

37. Zhao R, Song Y, Wang Y, et al. PD-1/PD-L1 blockade rescue exhausted CD8+ T cells in gastrointestinal stromal tumours via the PI3K/Akt/mTOR signalling pathway. Cell Prolif 2019;52:e12571.

38. Qiu XY, Hu DX, Chen WQ, et al. PD-L1 confers glioblastoma multiforme malignancy via Ras binding and Ras/Erk/EMT activation. Biochim Biophys Acta Mol Basis Dis 2018;1864:1754-69.

39. Ding L, Chen X, Xu X, et al. PARP1 Suppresses the 
Transcription of PD-L1 by Poly(ADP-Ribosyl)ating STAT3. Cancer Immunol Res 2019;7:136-49.

40. El-Khoueiry AB, Sangro B, Yau T, et al. Nivolumab in patients with advanced hepatocellular carcinoma (CheckMate 040): an open-label, non-comparative, phase 1/2 dose escalation and expansion trial. Lancet 2017;389:2492-502.

(English Language Editors: J. Gray and J. Chapnick)

Cite this article as: Huang X, Qin F, Meng Q, Dong M. Protein tyrosine phosphatase receptor type D (PTPRD)mediated signaling pathways for the potential treatment of hepatocellular carcinoma: a narrative review. Ann Transl Med 2020;8(18):1192. doi: 10.21037/atm-20-4733 